\title{
Intra-Vehicle Ranging in Ultra-Wide and Millimeter Wave Bands
}

\author{
Ales Prokes, Tomas Mikulasek, \\ Jiri Blumenstein \\ Department of Radio Electronic \\ Brno University of Technology \\ Brno, Czech Republic \\ prokes@feec.vutbr.cz
}

\author{
Christoph F. Mecklenbrauker \\ Institute of Telecommunications \\ Vienna University of Technology \\ Vienna, Austria
}

\author{
Thomas Zemen \\ Digital Safety \& Security Department \\ Austrian Institute of Technology $\mathrm{GmbH}$ \\ Vienna, Austria
}

\begin{abstract}
The paper deals with intra-vehicle ranging based on the time of arrival technique. The aim of the presented work is to compare the ranging accuracy in the 3 to $11 \mathrm{GHz}$ ultra-wide band and the 56 to $64 \mathrm{GHz}$ millimeter wave band. The comparison is performed using channel impulse responses calculated from complex transfer functions using a windowed IFFT. The complex transfer functions are measured by a four port vector network analyzer for empty and occupied car scenarios. The obtained results are discussed and differences in ranging accuracy between both bands are explained by means of several reference measurements carried out in an anechoic chamber.
\end{abstract}

Keywords-UWB; millimeter wave; ranging; positioning; localization; TOA

\section{INTRODUCTION}

There are several reasons why vehicle manufacturers are interested in intra-vehicle ranging and localization. They are going to implement WLAN into vehicles in order to allow passengers to share multimedia data or access the Internet. Localization of mobile multimedia devices within the car compartment allows to optimize the radiation pattern of access point antenna arrays and to reduce the radiated power. The other possible application of intra-car localization results in safeguarding the start of an engine. There are several techniques under development preventing the undesired start of the engine. One of them localizes the car key and allows to start the engine only if the key is within the designated area (e.g. in the pocket of a driver) inside the vehicle.

Localization can be implemented in three- or twodimensional space using the triangulation or the trilateration technique respectively [1]. Measuring the distance is often based on the time-of-arrival (TOA) technique which offers excellent time resolution and multipath components (MPC) separation in the case of a large system bandwidth. The 3.1 to 10.6 GHz ultra-wide band (UWB) and 57 to $64 \mathrm{GHz}$ millimeter wave (MMW) band [2], [3] are the most promising candidates for such applications. The large amount of work that has been previously published deals with channel sounding in the time domain, where some advanced techniques such as matched filtering or deconvolution of known very narrow (often Gaussian) pulses [4]-[6] or well-correlated binary sequences [1], [7] can be used. An example of a TOA based system with excellent time resolution using a maximal length binary sequence for time domain channel sounding is presented in [2]. However, for the above mentioned applications, only rough distance estimation is sufficient.

For the purpose of comparing both the UWB and MMW band ranging accuracies, our analysis is based on the channel impulse response (CIR) calculated from the measured complex transfer function (scattering parameter $S_{21}$ referred to as the forward voltage gain) using a windowed inverse fast Fourier transform (IFFT) [9]-[10].

The main contribution of the paper is statistic evaluation of the ranging accuracy in both bands carried out for two different measurement scenarios (empty and occupied car) and for original and interpolated CIR. In order to understand some differences in the calculated statistics we carried out a couple of measurements with the same setup in an anechoic chamber. We analyzed the effect of beam shadowing by a car seat and by a person and also examined the reflection properties of the side window and of various metallic shapes.

The rest of the paper is organized as follows. Section 2 describes the measurement setup including the antenna characteristic and ranging limitations. Section 3 briefly informs about the techniques of signal processing used for calculating the distance. Then, in the most important section, Section 4, the statistics of ranging accuracy and the comparison of both bands are given. The differences in statistics are explained in Section 5. The summary of the paper is given in the Conclusion.

\section{MEASUREMENT SETUP}

The block diagrams of the measurement setups for the UWB and MMW band are shown in Fig. 1. The complex channel transfer functions corresponding to the $S_{41}, S_{42}$, and $S_{43}$ scattering parameters are measured in the case of UWB by the four port vector network analyzer (VNA) Agilent Technologies E5071C while the scattering parameter $s_{21}$ in the MMW band is measured by the four ports VNA Rohde \& Schwarz ZVA67. Due to large attenuation in the coaxial cables and large propagation loss in the MMW band, the power amplifier (PA) Quinstar QPW-50662330 is used to extend the measuring system dynamic range. That is why we are able to measure only one MMW transfer function per frequency sweep. The output power $P_{\text {out }}$ of both VNAs is set to $5 \mathrm{dBm}$ and 
a)

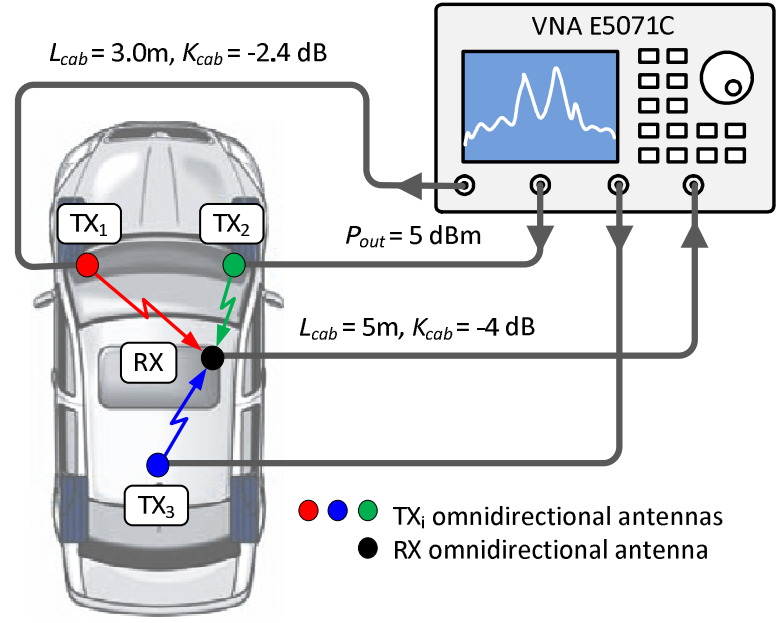

b)

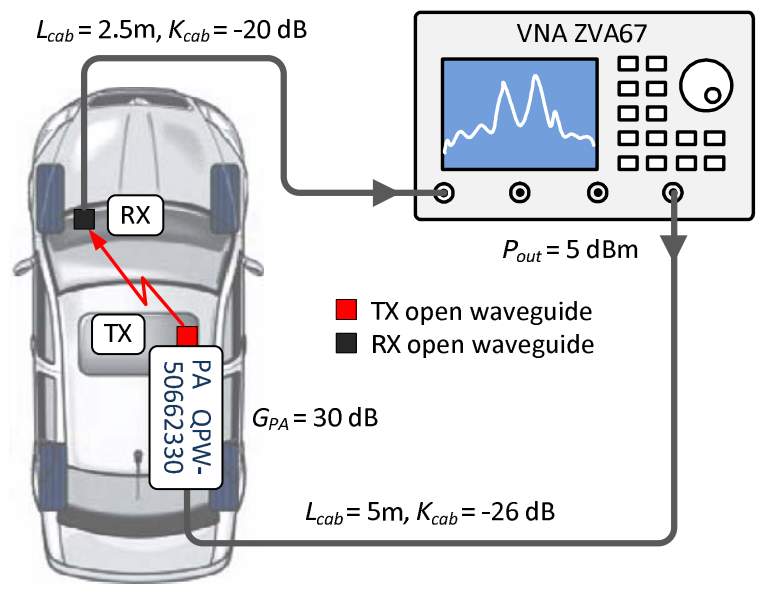

Fig. 1. The measurement setups for channel sounding in the a) UWB and b) MMW band ( $L_{c a b}$ is the coaxial cable length, $K_{c a b}$ is the measured cable transmission, $G_{P A}$ is the PA gain, and $P_{\text {out }}$ is the VNA output power).

the resolution bandwidth used for all measurements is chosen as $100 \mathrm{~Hz}$. For the purpose of comparison, we set the same bandwidth, $8 \mathrm{GHz}$, in both the UWB (3 to $11 \mathrm{GHz}$ ) and MMW ( 56 to $64 \mathrm{GHz}$ ) band and the same frequency step of $10 \mathrm{MHz}$ which provides 801 measured frequency points. We use monocone antennas in UWB and open waveguides in the MMW band, which are connected to the VNA by phase stable coaxial cables. It is obvious from Fig. 2, that both the UWB antenna and open waveguide exhibit noticeable gain dependence on the elevation angle (except the H-plane of the mono-cone antenna). Therefore the antennas and waveguides were mutually placed out of their minimum radiation region. The measurement was carried out in a Skoda Octavia III 1.8 TSI car.

\section{A. Antenna Placement}

The antenna positions for measurements in UWB and MMW band were very similar. In order to analyze the ranging accuracy for real possible antenna locations and for the scenario involving both the line-of-sight (LOS) and no line-ofsight (NLOS) wave propagation, we situated, as shown in Fig. 3, the transmitting (TX) UWB antennas on the left and right side of the dash-board, top corners of the windshield and
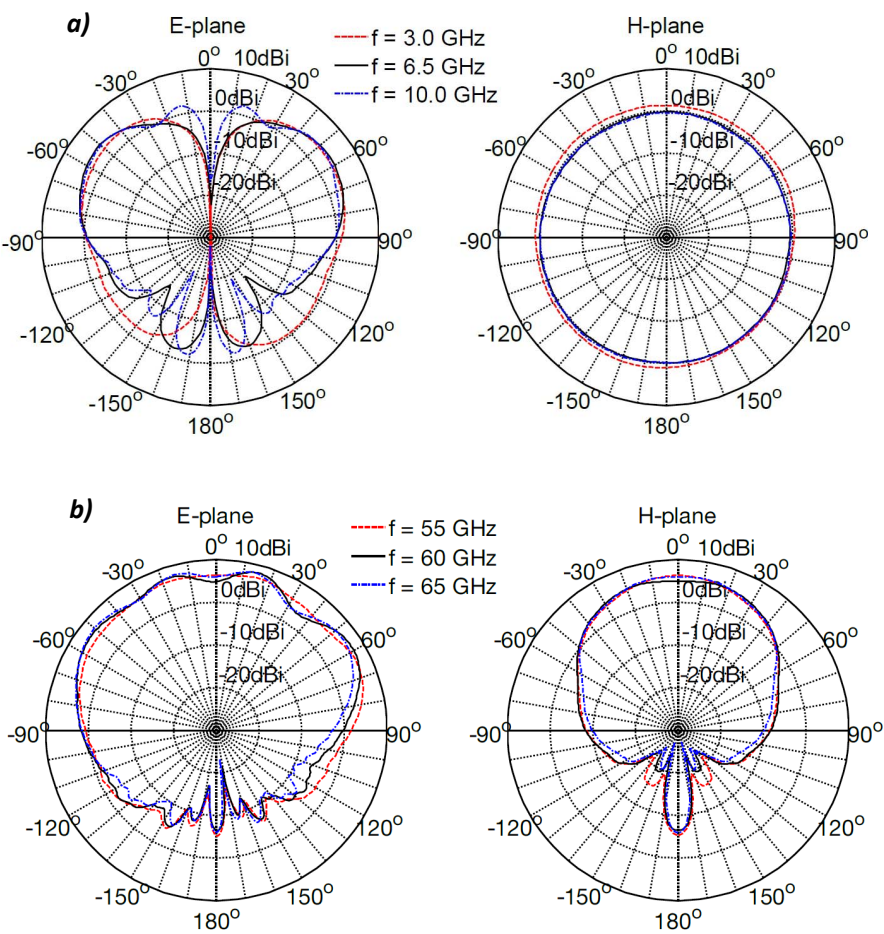

Fig. 2. Measured radiation patterns of a) the mono-cone UWB antenna and b) the open waveguide.

at the rear part of the ceiling. We then placed successively the receiving antenna, $(\mathrm{RX})$ mounted on a tripod, on all seats.

In the case of the MMW measurement we interchanged the RX and TX antenna positions due to large transmitter dimensions (see Fig. 4). In all the measurements in UWB there was at least one NLOS antenna pair. Therefore we evaluated the accuracy for the two different scenarios:

1. with one or two passengers sitting on the seats,

2. without passengers.

In both of these scenarios, both the LOS and NLOS wave propagations were present. The NLOS propagation was caused by the backrest of the seats and by the persons sitting inside the vehicle (only in the $2^{\text {nd }}$ case).

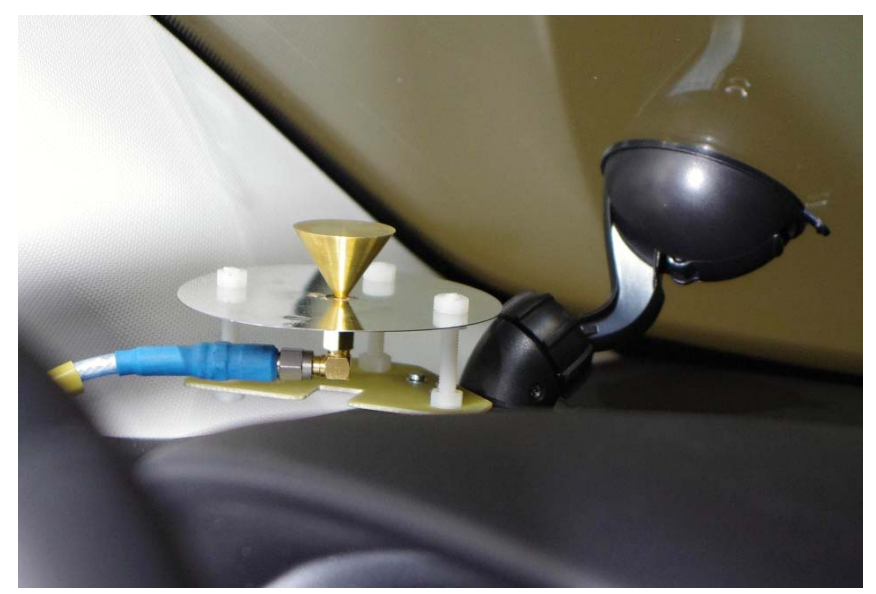

Fig. 3. Mono-cone UWB antenna with holder and suction cup above the dashboard. 


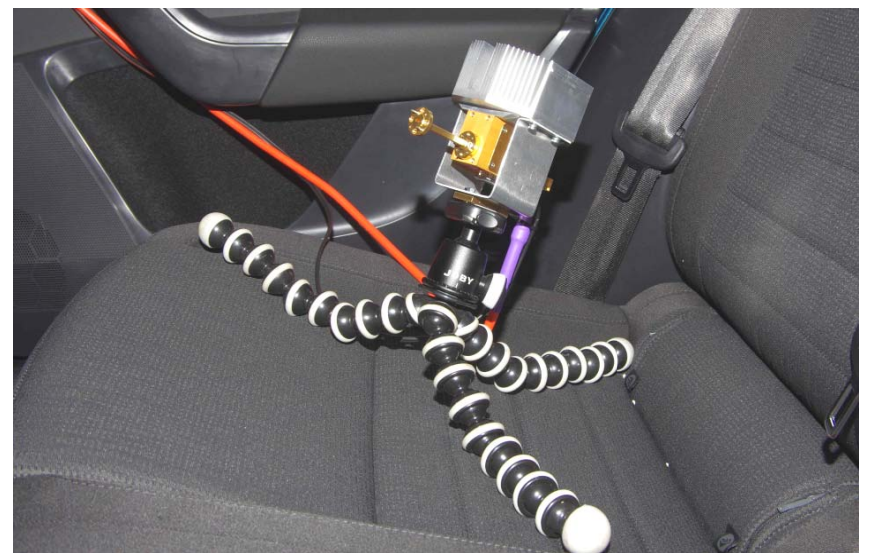

Fig. 4. MMW transmitter with PA Quinstar QPW-50662330 mounted on a photographic tripod (JOBY GorillaPod).

\section{B. Ranging Limitations}

The measuring system described above introduces some limitations of the ranging evaluation. As stated in [9] the bandwidth $B=f_{u}-f_{l}$, where $f_{u}$ is the upper frequency and $f_{l}$ is the lower frequency of the band, determines the distance resolution

$$
D_{r}=c T_{r}=\frac{c}{B}
$$

where $c=2,99 \cdot 10^{8} \mathrm{~m} / \mathrm{s}$ is the speed of the light, and $T_{r}=1 / B$ is the time resolution. The maximum measurable propagation distance depends on the number of measured frequency points $N$ inside the frequency band

$$
D_{\max }=\frac{c}{B} N=\frac{c}{f_{s}},
$$

where $f_{s}$ is the frequency step. It is obvious from (1) and (2) that narrowing the bandwidth, decreases the distance resolution and the reducing the measured points shortens the measurable propagation distance.

\section{Distance CALCUlATION}

The distance evaluation from the channel transfer function comprises three main steps. In the first step, the CIR is calculated using an N-point windowed IFFT. In the second step, the first arriving ray (the first peak of the CIR) is detected. The last step then consists of propagation time and the corresponding distance calculation.

The IFFT windowing is used to reduce the undesirable effects related to time domain leakage. After some experiments, we selected the Blackmann window because it ensures high side lobes suppression of $58 \mathrm{~dB}$ regarding to the main lobe (vs. $43 \mathrm{~dB}$ of the Hamming window or $47 \mathrm{~dB}$ of the Hanning window), high side lobe fall off, and reasonable main lobe width of 1.68 bins (vs. 1.3 bins of the Hamming window or 1.2 bins of the Hanning window) for $3 \mathrm{~dB}$ drop [9]. High side lobes suppression is preferred to main lobe width, because it decreases the probability of unwanted detection of a side lobe considered to be the first arriving ray.

The peak detecting algorithm is based on the threshold calculation, detecting the first value above the threshold and searching for the first peak behind the threshold crossing. The threshold level is generally determined by the noise floor. Its value is equal to the noise peak level, i.e. to the maximum amplitude of the CIR where the multipath component amplitudes are below noise. The time intervals used for the noise floor estimation were $0-1.25$ ns (i.e. before receiving the first ray) and 90 - 100 ns (where the MPC can be neglected). These time intervals correspond to the distances $0-37.5 \mathrm{~cm}$ (the minimum distance of RX-TX antennas in all scenarios is $50 \mathrm{~cm}$ ) and $27-30 \mathrm{~m}$. Note that according to (2), $30 \mathrm{~m}$ is the maximum measurable propagation distance. It is obvious that the proposed algorithm works well in both the LOS and NLOS scenarios but it fails in some NLOS cases when the first (direct) ray is drowned into the noise due to strong attenuation caused by obstacles (by passengers).

The distance of RX and TX antennas is given by the formula $D_{A}=c T_{D}$, where $T_{D}$ is the arrival time of the first detected ray.

\section{RANGING STATISTICS}

As mentioned in chapter II $A$, the ranging error statistics were calculated separately for the empty and the occupied car. It was experimentally discovered that the two or three passengers sitting in the car compartment cause similar results. Hence we evaluated both these cases together. The number of CIRs used for ranging statistics calculation is 54 for UWB and 22 for MMW (approximately half of them were measured for the LOS scenario and half for the NLOS scenario).

According to (1), the distance resolution for both bands is $D_{r}=3.75 \mathrm{~cm}$. Because this resolution is relatively low, we also interpolated the CIR by a factor of 8 in order to increase the first peak detection accuracy $\left(D_{r}=0.47 \mathrm{~cm}\right)$. The reference antenna distances were measured by a ruler. The average error and standard deviation of ranging in an empty and occupied car are summarized in Table I and Table II. An example of the CIR magnitude, interpolated CIR magnitude, threshold and peak detection is shown in Fig. 5.

TABLE I. AVERAGE ERROR AND STANDARD DEVIATION OF RANGING IN UWB

\begin{tabular}{llcc}
\hline \multicolumn{1}{c}{ Scenario } & $\begin{array}{c}\text { Original } \\
\text { MMW }\end{array}$ & $\begin{array}{c}\text { Interpolated } \\
\text { MMW }\end{array}$ \\
\hline $\begin{array}{l}\text { Average error } \\
(\mathbf{c m})\end{array}$ & without passengers & 1.74 & 1.44 \\
\cline { 2 - 4 } & with passengers & 2,55 & 2.26 \\
\hline $\begin{array}{l}\text { Standard } \\
\text { deviation }(\mathrm{cm})\end{array}$ & without passengers & 3.94 & 3,65 \\
\cline { 2 - 4 } & with passengers & 6.46 & 6.21 \\
\hline
\end{tabular}

TABLE II. AVERAGE ERROR AND STANDARD DEVIATION OF RANGING IN MMW

\begin{tabular}{llcc}
\hline & \multicolumn{1}{c}{ Scenario } & $\begin{array}{c}\text { Original } \\
\text { MMW }\end{array}$ & $\begin{array}{c}\text { Interpolated } \\
\text { MMW }\end{array}$ \\
\hline $\begin{array}{llcc}\text { Average error } \\
(\mathbf{c m})\end{array}$ & without passengers & 1.11 & 0.73 \\
\cline { 2 - 4 } & with passengers & 6.10 & 6.67 \\
\hline $\begin{array}{l}\text { Standard } \\
\text { deviation (cm) }\end{array}$ & without passengers & 2.41 & 2.04 \\
\cline { 2 - 4 } & with passengers & 9.77 & 9.77 \\
\hline
\end{tabular}




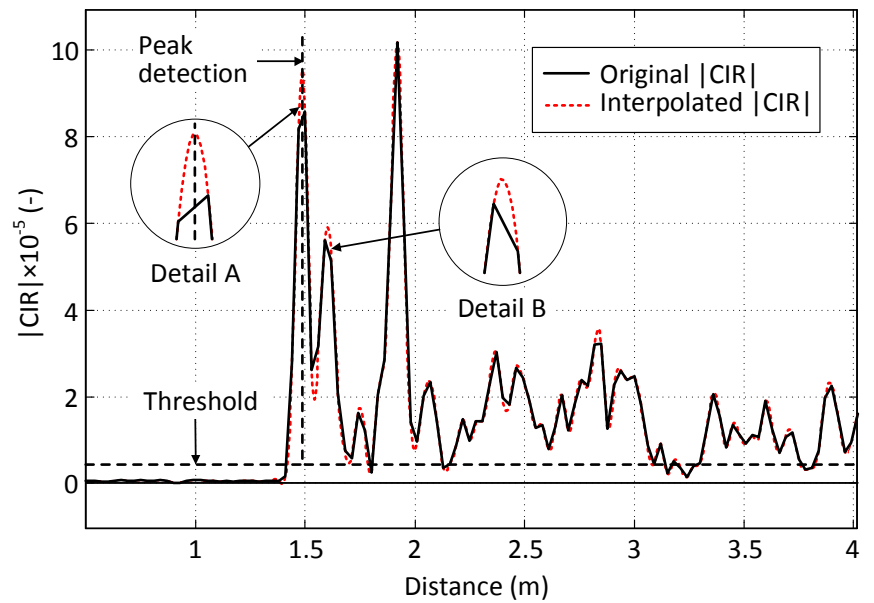

Fig. 5. An example of MMW CIR magnitude, interpolated MMW CIR magnitude, threshold and peak detection.

It is evident that both the UWB and MMW bands exhibit comparable ranging accuracy in an empty car but the situation in an occupied car is different. Both the average error and the standard deviation in the MMW band is much higher that of the UWB. It can also be observed that interpolation decreases slightly the average ranging error. As show the magnified details of the first and second peaks in Fig. 5, there could be differences in the position of the maximum value of the original CIR magnitude peak and interpolated CIR magnitude peak. It is obvious that the difference in the first peak detection can be sometimes positive (detail A) and sometimes negative (detail B). The average error then approaches the interpolated CIR magnitude ranging error. Therefore the difference in both the original and interpolated cases is relatively small especially when we take into account that the reference measurements with the ruler also exhibit indispensable error particularly in the NLOS scenario (it is e.g. impossible to measure accurately the antenna distance through the seat).

As mentioned in [9], the calculated distances exhibit a positive bias caused by a few phenomena. Compared to [9] in our last measurement, we excluded the effect of coaxial interfaces of the antennas which were not included during
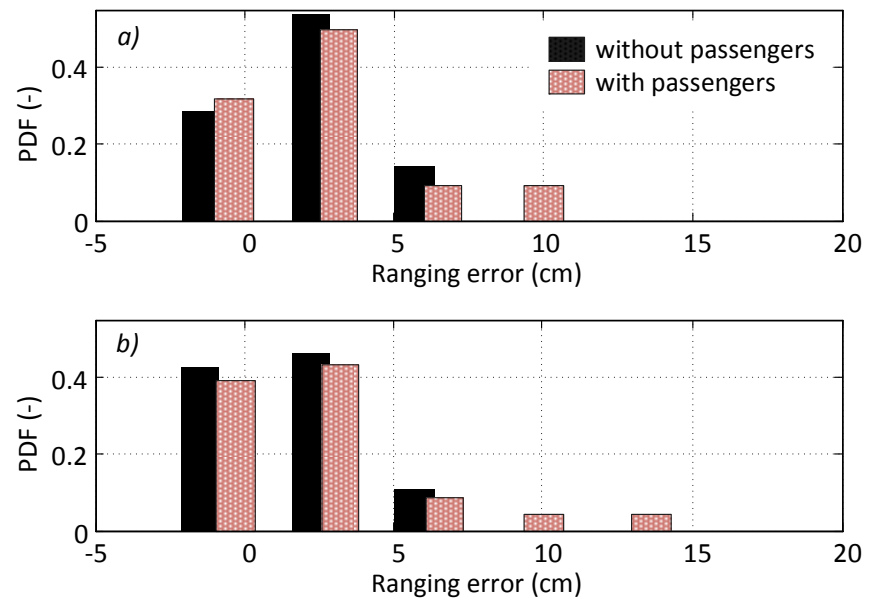

Fig. 6. Probability distribution function of ranging error in UWB for empty and occupied car calculated for a) original CIR and b) interpolated CIR.
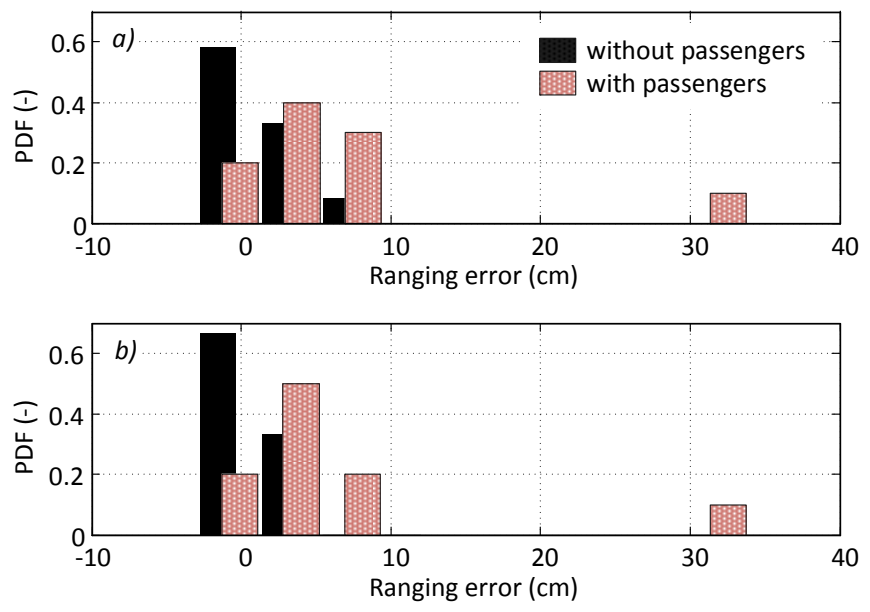

Fig. 7. Probability distribution function of ranging error in MMW band for empty and occupied car calculated for a) original and b) interpolated CIR.

calibration (we subtracted a distance corresponding to the length of a line between the connector and phase center of the antenna). We also measured the reference distances more precisely (between phase centers of antennas). Thus the positive bias is caused dominantly by a

- detection of some MPC traveling on a longer path instead of a direct ray when it is strongly attenuated in the NLOS propagation,

- lower velocity of an electromagnetic wave penetrating an obstacle.

Normalized histograms of the ranging errors corresponding to the probability distribution functions (PDF) calculated for both bands are plotted in Fig. 6 and Fig. 7. The figures demonstrate effects of the above phenomena causing spread of ranging errors towards to positive values. The effect of detection of a reflected MPC is obvious especially in the case of occupied car (with passengers) where the ranging error exceeds $10 \mathrm{~cm}$ in the UWB and $30 \mathrm{~cm}$ in the MMW band. The positive effect of interpolation on the ranging accuracy is evident in the case of empty car. When the car is occupied there are another ranging error resources and the interpolation effect does not approve itself as can be seen in Fig 7 a) and b).

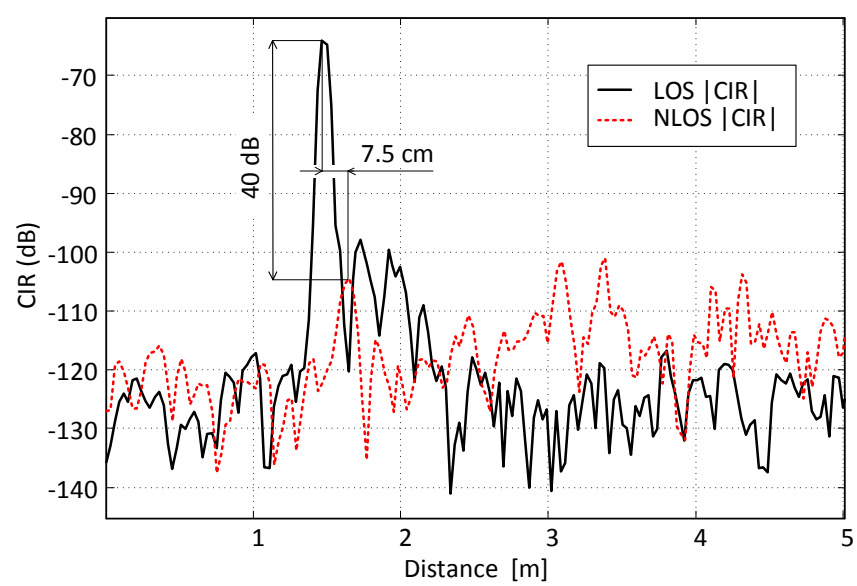

Fig. 8. Effect of shadowing by a human body (NLOS CIR) related to free space propagation (LOS CIR) in MMW band. 
However, as shown in Fig. 5, there are positive or negative differences in the peak positions of the interpolated and original CIRs. The negative differences cause e.g. occurrence of an error component at position of about $13 \mathrm{~cm}$ in Fig. $6 \mathrm{~b}$ ).

\section{REFERENCE MEASUREMENT IN ANECHOIC CHAMBER}

In order to understand the difference in the above statistics, and the ranging errors distribution we carried out a few measurements in the anechoic chamber. We investigated the effect of shadowing caused by a seat backrest and by a human body. The effect of shadowing in the MMW by a person standing in the middle between antennas is shown in Fig. 8, where the CIRs with person (marked as the NLOS CIR) and without person (marked as the LOS CIR) are plotted together. The RX to TX antenna distance was $145 \mathrm{~cm}$. In order to show a large shadowing effect, the CIR was plotted in $\mathrm{dB}$ scale. It is obvious that both the time delay and attenuation caused by a human body are relatively large and depend on the distance from the TX antenna to the person. The physical nature of the effect caused by a human body is probably ray diffraction because the depth of wave penetration into muscles in both bands is very small (below one centimeter for a power density decrease by a factor of $e^{-2}$, i.e. for $8.7 \mathrm{~dB}$ attenuation) [12]. Results for both bands are summarized in Table III. It is clear that the MMW band exhibits significantly larger attenuation $(A)$ and distance extension $(\Delta L)$ in the case of shadowing by a human body. This fact provides the reason for the larger average error in the real condition (see Table I and Table II). Note that the larger attenuation in case the body is close to MMW TX can cause also narrower radiation pattern of the open waveguide. The seat attenuation is larger in the UWB but the distance extension is very similar in both bands. An example of incorrect peak detection in the MMW band is shown in Fig. 7. Extremely strong attenuation due to the body attenuation causes detection of the reflected MPC travelling on a markedly longer path which results in more than $30 \mathrm{~cm}$ error.

We also checked the reflection properties of some materials and shapes. The reflection characteristics are generally very sensitive to the reflecting object position and distance related to the antennas. However, among the most interesting findings belong the reflective properties of the car windows (glass). The attenuation of MPCs related to the direct LOS ray is $12.2 \mathrm{~dB}$ in UWB and $34 \mathrm{~dB}$ in the MMW band (the MPC propagation distance was $269 \mathrm{~cm}$ while the direct ray propagated over a distance of $145 \mathrm{~cm}$ ) and is comparable, for example, to the reflection properties of a metal plain plate with dimensions of approximately $10 \times 10 \mathrm{~cm}$.

TABLE III. EfFect of Shadowing Related to Free Space Propagation

\begin{tabular}{|c|c|c|c|c|}
\hline \multirow[b]{2}{*}{ Scenario } & \multicolumn{2}{|c|}{ UWB } & \multicolumn{2}{|c|}{ MMW } \\
\hline & $\begin{array}{l}A^{\mathrm{a}} \\
\text { (dB) }\end{array}$ & $\begin{array}{l}\Delta L^{\mathrm{a}} \\
(\mathbf{c m})\end{array}$ & $\begin{array}{c}A \\
\text { (dB) }\end{array}$ & $\Delta L(\mathrm{~cm})$ \\
\hline Body in the middle & 19 & 3.2 & 40 & 7.5 \\
\hline Body close to TX & 20 & 4.1 & 53 & 11.0 \\
\hline Seat in the middle & 0.8 & 0.8 & 4.8 & 0.6 \\
\hline Seat close TX & 0.9 & 1.6 & 4.8 & 0.5 \\
\hline Glass from car window & 3 & 0.3 & 1.3 & 0.8 \\
\hline
\end{tabular}

\footnotetext{
${ }^{\mathrm{a}} A$ and $\Delta L$ stand for the attenuation and distance extension respectively
}

\section{CONCLUSION}

We carried out an extensive UWB and MMW measurement campaign for a vehicular passenger compartment. We compared the ranging accuracy in both bands and improved it in the UWB when compared to [9]. We also checked influence of interpolation of CIR on the ranging accuracy and we found that it take positive effect mainly in the case of empty car. We also explained the difference in the calculated statistics. For this purpose, we performed a couple of measurements in an anechoic chamber. Generally, we found that the ranging accuracy is very similar in both bands when the car is empty but the presence of passengers can markedly decrease the ranging performance especially in the MMW band.

Selected files of measured data are downloadable from the web page http://www.radio.feec.vutbr.cz/GACR-13-38735S/.

\section{ACKNOWLEDGMENT}

The research described in this paper was financed by the Czech Science Foundation, Project No. 13-38735S, and by National Sustainability Program under grant LO1401. For the research, infrastructure of the SIX Center was used.

\section{REFERENCES}

[1] Z. Sahinoglu, S. Gezici, and I. Guvenc, Ultra-wideband positioning systems, Cambridge UK: Cambridge University Press, 2008, vol. 2.

[2] M. G. Di Benedetto, T. Kaiser, A. F. Molisch, I. Oppermann, C. Politano, and D. Eds. Porcino, UWB Communications Systems: A Comprehensive Overview. New York: Hindawi, 2006.

[3] Working Group Spectrum Engineering, Use Of The $57-64 \mathrm{GHz}$ Frequency Band For Point-To-Point Fixed Wireless Systems. ECC Recommendation (09)01.

[4] T. C. K. Liu, D. I. Kim, and R. G. Vaughan, "A high-resolution, multitemplate deconvolution algorithm for time-domain UWB channel characterization,” Can. J. Elect. Comput. Eng., vol. 32, no. 4, pp. 20713, 2007.

[5] W. Niu, J. Li, T. Talty, "Ultra-wideband channel modeling for intravehicle environment," EURASIP J Wirel Commun Net, vol. 2009, Article ID 806209, pp. 1-12, 2009.

[6] A. Chandra, J. Blumenstein, T. Mikulášek, R. Marsalek, A. Prokeš, T. Zemen, Ch. Mecklenbräuker. "Use of CLEAN in Time-Domain Ultra Wide Band Channel Sounding for Intra Vehicular Environments," IET Intell. Transp. Sy., pp. 1-12, 2015.

[7] R. Zetik, M. Kmec, J. Sachs, and R. S. Thomä, "Real-Time MIMO Channel Sounder for Emulation of Distributed Ultrawideband Systems", Int. J. Antennas Propag, vol. 2014, Article ID 317683, pp. 1-16, 2014.

[8] R. Nakamura, and A. Kajiwara, "Empirical Study on $60 \mathrm{GHz}$ In-vehicle Radio Channel," Proc. of 2012 IEEE Radio and Wireless Symposium, Santa Clara, USA, pp.327-330, 2012.

[9] J. Blumenstein, A. Prokeš, T. Mikulášek, R. Maršálek, C. Mecklenbräuker, "Measurements of Ultra Wide Band In-vehicle channel - statistical description and TOA positioning feasibility study," EURASIP J Wirel. Comm., Springer, pp. 1-10, 2015.

[10] J. Blumenstein, T. Mikulášek, R. Maršálek, A. Prokeš, T. Zemen, C. Mecklenbräuker, "In-vehicle mm- Wave Channel Model and Measurement," In Proc. of IEEE 80th Vehicular Technology Conference (VTC Fall). Vancouver, Canada, pp. 1-5, 2014,

[11] F. J. Harris, "On the Use of Windows for Harmonic Analysis with the Discrete Fourier Transform," Proc. of the IEEE, vol. 66, no. 1, pp. 5183, 1978.

[12] C. C. Johnson, and A. W Guy, "Nonionizing electromagnetic wave effects in biological materials and systems," Proc. of IEEE, vol. 60, no. 6, pp. $692-718,1972$. 